\title{
Reoperação em pacientes revascularizados
}

Ibraim Masciarelli F. PINTO*, Leopoldo S. PIEGAS*, Luiz Alberto P. MATTOS*, Luiz Fernando L. TANAJURA*, Enilton T. EGITO*, Camilo Abdulmassih NETO*, Antoninho S. ARNONI*, Luiz Carlos Bento de SOUZA*, Adib D. JATENE*, J. Eduardo M. R. SOUSA*

PINTO, I. M. F.; PIEGAS, L. S.; MATTOS, L. A. P.; TANAJURA, L. F. L.; EGITO, E. T.; ABDULMASSIH NETO, C.; ARNONI, A. S.; SOUZA, L. C. B.; JATENE, A. D.; SOUSA, J. E. M. R. - Reoperação em pacientes revascularizados. Rev. Bras. Cir. Cardiovasc., 2(3): 171-174, 1987.

RESUMO: Um grupo de pacientes submetidos a cirurgia de revascularizaçâo do miocárdio pode vir a necessitar uma reoperação tardia. Neste trabalho, discute-se quais os possiveis fatores determinantes deste evento, bem como o prognóstico imediato destes indivíduos. Foram analisados $261(10,5 \%)$ pacientes, submetidos a reoperação para revascularização isolada do miocárdio entre janeiro de 1984 e junho de 1986. Havia $109(41 \%)$ hipertensos e $145(55 \%)$ portadores de infarto do miocárdio prévio. A reoperação foi indicada pela presença de lesōes no leito nativo das artérias em $66(25 \%)$, por lesōes nos enxertos em $88(33 \%)$ e por lesōes nos leitos nativos e nos enxertos em $107(42 \%)$. O tempo médio de reoperação foi de $7,1 \pm 3,3$ anos, sendo $75 \%$ dos pacientes reoperados num intervalo superior a 6 anos. 0 número global de artérias mamárias obstruídas foi de $5(20 \%)$ enquanto que $171(75 \%)$ das pontes de veia safena apresentavam lesōes. A mortalidade hospitalar foi de $23(9 \%)$. Os autores discutem as relaçōes destes achados com a incidência da reoperação bem como as possiveis causas da mortalidade elevada.

DESCRITORES: miocárdio, revascularização, cirurgia.

\section{INTRODUÇÃO}

A cirurgia de revascularização do miocárdio, seja com o uso de pontes de veia safena, ou com anastomose de artéria mamária interna, é a forma ideal de tratamento da insuficiência coronária, em grande número de pacientes.

Durante a evolução tardia, porém, um grupo de indivíduos revascularizados pode vir a necessitar de uma nova cirurgia, devido ao aparecimento de lesōes ateroscleróticas nos enxertos utilizados, ou no leito nativo de artérias não previamente tratadas ${ }^{4,8}, 13$.

Este trabalho teve por objetivo analisar as principais características de um grupo de pacientes consecutivamente reoperados, tentando determinar quais os principais fatores que levaram a esta situaçāo, bem como sua evolução imediata.

\section{MATERIAL E MÉTODOS}

Entre janeiro de 1984 e junho de 1986, foram realizadas 2488 cirurgias de revascularização do miocárdio, isoladas. Dentre estas, $261(10,5 \%)$ foram reoperaçōes e compōem a casuística analisada.

As características clínicas do grupo estudado encontram-se na Tabela 1.

Tabela 1

CARACTERISTICAS CLINICAS

\begin{tabular}{|c|c|}
\hline • Sexo M & $\begin{array}{l}3 \pm 5 \text { anos } \\
222(85 \%)\end{array}$ \\
\hline - $\quad \mathrm{F} \ldots$ & $39(15 \%)$ \\
\hline • Hipertensāo ....... & $109(41 \%)$ \\
\hline 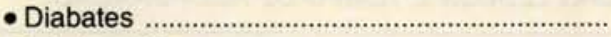 & $44(17 \%)$ \\
\hline • Infarto prévio & $145(55 \%)$ \\
\hline
\end{tabular}

Trabalho realizado no Instituto Dante Pazzanese de Cardiologia. São Paulo, SP, Brasil.

Apresentado ao 14: Congresso Nacional de Cirurgia Cardiaca. Salvador, BA, 27 e 28 de março, 1987

- Do Instituto Dante Pazzanese de Cardiologia.

Endereço para separatas: Ibraim M. F. Pinto. Caixa Postal 215, 01000 São Paulo, SP, Brasil. 
PINTO, I. M. F.; PIEGAS, L. S.; MATTOS, L. A. P.; TANAJURA, L. F. L.; EGITO, E. T.; ABDULMASSIH NETO, C.: ARNONI,

A. S.; SOUZA, L. C. B.; JATENE, A. D.; SOUSA, J. E. M. R. - Reoperação em pacientes revascularizados. Rev. Bras. Cir. Cardiovasc., 2(3): 171-174, 1987.

Na primeira cirurgia, $46(17 \%)$ pacientes foram operados para tratar um vaso, $122(47 \%)$ para tratar 2 vasos, $76(30 \%)$ para tratar 3 vasos e $16(6 \%)$ para tratar 4 vasos. Em $9(3 \%)$ casos, usou-se anastomose de artéria mamária interna esquerda isolada, em 24 (9\%) empregou-se anastomose mamária interna associada a pontes de veia safena e, em $228(88 \%)$, utilizaram-se pontes de veia safena isoladas. $O$ número médio de enxertos por paciente foi de 2,3 .

O intervalo de tempo entre a primeira e a segunda cirurgias variou de 2 meses a 16 anos, sendo de notar que $194(75 \%)$ pacientes foram reoperados com intervalo de tempo igual ou superior a 6 anos (Tabela 2).

TABELA 2

INTERVALO ENTRE AS CIRURGIAS

\begin{tabular}{|c|c|c|}
\hline \multicolumn{2}{|c|}{ TEMPO } & \multirow{2}{*}{$\frac{N^{\circ}}{15(05 \%)}$} \\
\hline$<$ & 1 ano & \\
\hline $1-$ & 3 anos & $17(07 \%)$ \\
\hline $3-$ & 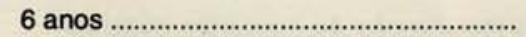 & $40(15 \%)$ \\
\hline $6-$ & 9 anos & $106(40 \%)$ \\
\hline $10-$ & 13 anos & $58(23 \%)$ \\
\hline $13-$ & 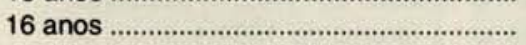 & $25(10 \%)$ \\
\hline
\end{tabular}

Procurando-se correlacionar o total de cirurgias de revascularização miocárdica isolada praticadas no Serviço, em um determinado ano, e as reoperaçōes que tardiamente se fizeram necessárias, encontraram-se os seguintes números: em 1977, realizaram-se 550 cirurgias, com 25 (5\%) de reoperaçōes tardias; em 1978, 601 cirurgias e 31 (5\%) reoperações; em 1979, 626 revascularizaçōes e $15(2 \%)$ reoperaçōes; em 1980, 746 revascularizaçōes e $24(3 \%)$ reoperaçōes.

A reoperação foi indicada pela presença de progressão de lesões no leito nativo em 66 (25\%), por lesōes tardias nos enxertos usados na primeira cirurgia em 88 $(33 \%)$ e por lesões, tanto no leito nativo como nos enxertos, em 107 (42\%). Dos 195 pacientes que exibiam lesōes no leito dos enxertos (isoladamente ou associadas a lesões nas artérias nativas), 186 (95\%) apresentavam obstruçōes nas pontes de veia safena e $9(5 \%)$ nas artérias mamárias.

O tempo médio de reoperação para cada subgrupo não variou (Tabela 3 ).

TABELA 3

LOCAL DAS LESOESS TEMPO DE REOPERAÇĀO

\begin{tabular}{lrc}
\hline LOCAL LESÃO & N. & TEMPO DE OPERAÇÃO \\
\hline Leito nativo & 66 & $7,1 \pm 3,2$ anos \\
Enxertos & 88 & $7,1 \pm 4,2$ anos \\
Ambos & 107 & $6,8 \pm 3,3$ anos \\
\hline
\end{tabular}

Na reoperação, $42(16 \%)$ pacientes receberam enxerto para apenas 1 vaso, $96(37 \%)$ para 2 vasos, 87 $(33 \%)$ para 3 vasos, $33(12 \%)$ para 4 vasos e $3(1 \%)$ para 5 vasos. Em 4 (2\%) pacientes, foram usadas anastomoses de artéria mamária isolada, $71(27 \%)$ receberam anastomoses de artéria mamária associada a pontes de veia safena e, em $186(71 \%)$, usaram-se pontes de veia safena isoladamente. $O$ número médio de enxertos por paciente foi de 2,5 .

A mortalidade hospitalar deste grupo foi de $23 / 261$ $(9 \%)$ enquanto que, no mesmo período, nos pacientes submetidos a revascularização do miocárdio como primeira cirurgia, a mortalidade hospitalar foi de $82 / 2227$ $(3,6 \%)$.

\section{COMENTÁRIOS}

A principal finalidade deste trabalho foi tentar identificar, retrospectivamente, as características dos pacientes que vieram a necessitar de uma reoperação de revascularização miocárdica isolada.

$\mathrm{Na}$ análise dos antecedentes pessoais dos pacientes reoperados, observou-se um número grande de portadores de hipertensão arterial sistêmica e de infarto do miocárdio prévio. Estes achados concordam com relatos da literatura ${ }^{2}, 4,9,12$, sugerindo que, neste grupo de pacientes operados, a progressão da doença aterosclerótica, tanto nos enxertos como no leito nativo das artérias coronárias, é maior.

O tempo médio de reoperação foi de $7,1 \pm 3,6$ anos, sendo que $75 \%$ das reoperaçōes ocorreram num intervalo de tempo igual ou superior a 6 anos. Nota-se que a incidência de reoperações é maior, conforme aumenta o intervalo de tempo entre as operaçōes. Tentando analisar este evento por um outro prisma, notou-se que o número de reoperaçōes foi maior nas séries cirúrgicas mais antigas $(3 \%$ em $1980,2 \%$ em $1979,5 \%$ em $1978,5 \%$ em 1977).

O tempo médio de reoperação não diferiu significativamente nas cirurgias desencadeadas pela progressāo de lesões no leito nativo, ou por lesões dos enxertos. Dados semelhantes são referidos na literatura ${ }^{1}, 3,5,6$. 10. 11. Este fato reforça a hipótese de que a aterosclerose mantém seu caráter de doença evolutiva, mesmo em pacientes tratados cirurgicamente.

Em relação ao tipo de enxerto usado na primeira cirurgia, observou-se que, dos 9 pacientes que receberam anastomose mamária interna isolada, 4 (44\%) tinham obstruçōes, enquanto que os $5(66 \%)$ restantes foram reoperados para revascularizaçāo de outras áreas. Quando a mamária foi utilizada em associaçāo com a ponte de veia safena (24 pacientes), 5 (20\%) tinham obstrução da mamária e os $19(80 \%)$ restantes, 
PINTO, I. M. F.; PIEGAS, L. S.; MATTOS, L. A. P.; TANAJURA, L. F. L.; EGITO, E. T.; ABDULMASSIH NETO, C.; ARNONI, A. S.; SOUZA, L. C. B.; JATENE, A. D.; SOUSA, J. E. M. R. - Reoperação em pacientes revascularizados. Rev. Bras. Cir. Cardiovasc., 2(3): 171-174, 1987.

lesōes na ponte de veia safena e/ou progressão da doença no leito nativo. Globalmente, encontraram-se, na reoperaçāo, 24 (73\%) mamárias pérvias e 9 (27\%) mamárias ocluidas. Dos 228 pacientes que, na primeira cirurgia, receberam apenas enxertos de veia safena, 171 (75\%) tinham oclusão da veia, enquanto que, em 57 $(25 \%)$, as veias safenas estavam integras e a revascularização foi realizada em outra área.

A análise destes números nos permite afirmar que a incidência de obstruçōes nas artérias mamárias é significantemente menor do que a incidência de obstruçōes nas pontes de safena.

Constatou-se, também, que, na segunda cirurgia, o enxerto com artérias mamárias foi mais utilizado. Isto se deve à tendência de utilizar este enxerto com maior freqüência nos anos mais recentes. Na reoperaçāo, a anastomose com artéria mamária interna foi utilizada em $75(28 \%)$ pacientes, sendo que 51 (68\%) em substituiçăo a pontes de veia safena lesadas.
A mortalidade hospitalar dos pacientes reoperados foi 2,5 vezes superior àquela dos submetidos a revascularizaçāo miocárdica como primeiro operação. Esta maior mortalidade também foi encontrada por outros grupos: CAMPEAU et alii 5\% ${ }^{4}$ e HALL et alii ${ }^{6}$, no Texas Heart Institute, $9 \%$. O fato pode ser explicado pela faixa etária mais elevada, pela presença significativa de hipertensos e infartados prévios e pela progressão da doença aterosclerótica.

Estes achados permitem concluir que: a faixa etária mais elevada, a hipertensão arterial sistêmica e a ocorrência de infarto prévio associaram-se à incidência de reoperaçāo; o número de vasos tratados nāo diferiu nas duas operações; o tempo de progressão da aterosclerose foi semelhante nas artérias nativas e nos enxertos; a taxa de reoperação é maior, na medida em que aumenta o intervalo após a primeira cirurgia; o número de artérias mamárias obstruídas foi inferior ao de veias safenas; a mortalidade hospitalar dos pacientes reoperados é elevada.

RBCCV $44205-37$

PINTO, I. M. F.; PIEGAS, L. S.; MATTOS, L. A. P.; TANAJURA, L. F. L.; EGITO, E. T.; ABDULMASSIH NETO, C.; ARNONI, A. S.; SOUZA, L. C. B.; JATENE, A. D.; SOUSA, J. E. M. R. - Second surgery for myocardial revascularization. Rev. Bras. Cir. Cardiovasc., 2(3): 171-174, 1987.

ABSTRACT: A second surgery for myocardial revascularization may be needed in some patients. One still discussed the reasons and the risk of such procedure. A group of 162 patients who underwent a second coronary by-pass graft surgery was analized. There was a significant number of patients with high blood pressure and with previous myocardial infarction. The reason for reoperation was either lesion within the by-pass lumen or a lesion within the lumen of a native artery non-previously treated. Most patients underwent the second coronary by-pass graft surgery after an interval of, at least, 6 years after the previous surgery. In-hospital mortality was $9 \%$ (23 patients). Authors discuss the possivle causal relationship between these findings and reoperation, as well as the causes of the high mortality.

DESCRIPTORS: myocardial revascularization, surgery.

\section{REFERÊNCIAS BIBLIOGRÁFICAS}

1 ARNONI, A. S.; PAULISTA, P. P.; SOUZA, L. C. B.; SOUSA, J. E. M. R.; FICHINO, M. Z. S.; ANGRISANI NETO, S.; BONATELLI FILHO, L.; JATENE, A. D. - Reoperação em cirurgia de revascularização do miocárdio. Arq. Bras. Cardiol., 41(4): 317-322, 1983.

2 BARBORIAK, J. J.; BARBORIAK, D. P.; ANDERSON, A. J.; RIMM, A. A.; TRISTANI, F. E.; FLEMMA, R. J. Risk factors in patients undergoing a second aorta-coronary bypass procedure. J. Thorac. Crdiovasc. Surg., 76(1): 111-114, 1978.

3 BRUMMET, C.; REVES, J. G.; LELL, W. A.; SMITH, L. R. - Patient care problems in patients undergoing reoperation for coronary artery grafting surgery. Can. Anaesth. Soc. J., 31: 213-220, 1984.
4 CAMPEAU, L.; ENJALBERT, M.; LESPÉRANCE, J.; BOURRASSA, M. G.; KWITEROVIC Jr., P.; WACHOLDER, S.; SNIDERMAN, A. - The relation of risk factors to the development of atherosclerosis in saphenousvein bypass grafts and the progression of disease in the native circulation: a study 10 years after coronary bypass surgery. N. Engl. J. Med., 311(21): 1329-1332, 1984.

5 FAVALORO, R. G. - Direct myocardial revascularization: a ten year journey. Am. J. Cardiol. 43(1): 109-129, 1979.

6 HALL, R. J.; ELAYDA, M. A. A.; GRAY, A. G.; COOLEY, D. A. - Reoperation for coronary artery disease. J. Am. Coll. Cardiol. 7(2): 32A, 1986. (Resumo)

7 KOBAYASHI, T.; MENDES, A. M.; ZUBIATE, P.; VANSTROM, N. R.; YOKOAMA, T.; KAY, J. H. - Repeat aortocoronary bypass grafting. Chest, 73(4): 446-449, 1978. 
PINTO, I. M. F.; PIEGAS, L. S.; MATTOS, L. A. P.; TANAJURA, L. F. L.; EGITO, E. T.; ABDULMASSIH NETO. C.; ARNONI. A. S.; SOUZA, L. C. B.; JATENE, A. D.; SOUSA, J. E. M. R. - Reoperaçāo em pacientes revascularizados. Rev. Bras. Cir. Cardiovasc., 2(3): 171-174, 1987.

8 KRAUSE Jr., A. H. ; PAGE, U. S.; BIGELOW, J. C.; OKIES, J. E.; DUNLAP, S. F. - Reoperation in symptomatic patients after direct coronary artery revascularization. J. Thorac. Cardiovasc. Surg., 75(4): 499-504, 1978.

9 LAMAS, G. A.; MUDGE Jr., G. H.; COLLINS Jr., J. J.; KOSTER, R.; COHN, L. H.; FLATLEY, M.; SHEMIN, R.; COOK, E. F.; GOLDMAN, L. - Clinical response to coronary artery reoperations. J. Am. Coll. Cardiol., 8(2): 274-279, 1986.

10 LITLE, B. W.; LOOP, F. D.; COSGROVE, D. M.; TAYLOR, P. C.; GOORMASTIC, M.; GILL, C. C.; GOLDING, L. A. R.; STEWART, R. V. - Fifteen hundred coronary reoperations: results and determinants of early and late survival. J. Am. Coll. Cardiol., 7(2): 31A, 1986. (Resumo)

11 QAZI, A.; GARCIA, J. M.; MISPIRETA, L. A.; CORSO, P. J. - Reoperation for coronary artery disease. Ann. Thorac. Surg., 32(1): 16-18, 1981.

12 REUL Jr., G. J.; COOLEY, D. A.; OTT, D. A.; COELHO, A.; CHAPA, L.; ETEROVIC, I. - Reoperation for recurrente coronary artery disease: causes, indications, and results in 168 patients. Arch. Surg., 114(11): 1269-1275, 1979.

13 WUKASCH, D. C.; TOSCANO, M.; COOLEY, D. A.; REUL Jr., G. J.; SANDIFORD, F. M.; KYGER III, E. R.; HALLMAN, F. L. - Reoperation following direct myocardial revascularization. Circulation, 56(Supl. 2):3-7, 1977.

\section{Discussão}

\section{DR. NEI ANTÔNIO REY Porto Alegre, RS}

Gostariamos de agradecer, à Comissão Organizadora, a honra de podermos comentar tão importante trabalho, e aos autres, pelo excelente material. Em primeiro lugar, desejamos enfatizar a importância do tema. Para isto, vamos nos valer de trabalho do mesmo grupo, publicado nos Arquivos Brasileiros de Cardiologia de outubro de 1983 , no qual o percentual de reoperaçóes foi de $2,4 \%$. Este número, no presente trabalho, já sobe para $10,5 \%$. Vemos, então, a importância crescente deste tema. Este percentual de reoperação pode ser mais alto, como o da Cleveland Clinic, que, no ano de 1986, atinge $14 \%$, ou mais baixo, como o do Hospital Nossa Senhora da Conceiçāo, no qual trabalhamos, e que, para 1986 , foi de 6,25 . O mais importante é tomarmos ciência de que, embora a mais moderna técnica e tática cirúrgicas hoje utilizadas para os doentes que estão sendo, agora, submetidos à revascularização do miocárdio, espera-se que $7 \%$ sofram reoperaçōes, nos próximos 10 anos. Analisando os números do trabalho agora apresentado, verificamos que $17 \%$ dos pacientes tiveram uma artéria trata- da e $47 \%$, duas. Juntos, somam $64 \%$ de pacientes que tiveram uma ou duas artérias tratadas. Por outro lado, $96 \%$ foram revascularizados previamente com veia safena e somente $4 \%$ com o uso de artéria mamária interna (AMI). A tendência de revasculizar poucas artérias e a não utilizaçāo da AMI foi uma tendência do passado, quando esses pacientes foram operados. Hoje, sabemos que estes fatores predispōem os pacientes à reoperação, o que o presente trabalho ajudou a demonstrar. A tendência atual da cirurgia de coronária é de que os pacientes tenham revascularizadas várias artérias, de que se faça revascularização completa e de que se usem as artérias mamárias, o que deverá reduzir o número de candidatos à reoperaão. Analisando o tempo médio de reoperação, que, no presente caso, foi de 7,1 anos, vê-se que foi importante a aterosclerose, determinando lesāo nos enxertos, ou no leito nativo das artérias, como agente principal e determinante das reoperaçōes. Isto faz com que um número maior de pacientes seja portador de lesão do tronco da coronária esquerda (LTCE), idade avançada e má função ventricular esquerda. Dentro desta contingência, acredito que a mortalidade de $9 \%$ apresentada seja perfeitamente aceitável. Ainda sobre o intervalo entre a primeira cirurgia e a reoperaçāo, com o maior uso da AMI, este deverá ser maior, nas futuras séries. Uma outra conclusão, a de que a HAS foi significativa nos casos reoperados, nós a atribuímos, também, ao menor emprego da AMI, já que os chamados fatores de risco: colesterol alto, fumo e HAS nāo costumam influir nos pacientes revasculrizados com a AMI. A esternotomia prévia não tem sido causa de lesão da $\mathrm{AMI}$; esta pode ser dissecada e seu uso é recomendável nas reoperações. Concluindo, queremos ressaltar que o uso da AMI e a revascularizaçāo completa são os fatores que mais influem para diminuir o número de reoperações.

\section{DR. IBRAIM PINTO \\ (Encerrando)}

Agradecemos o comentário do Dr. Nei Rey, com o qual estamos de pleno acordo, Todos os Serviços que se têm dedicado à revascularização do miocárdio têm observado um amento crescente no número de reoperaçōes. Esse aumento deve-se à progressão da aterosclerose coronária, e não a problemas de técnica cirúrgica. Por isso, a reintervenção costuma, na maioria dos casos, ser tardia. As artérias mamárias empregadas na revascularizaçāo miocárdica são bem mais resistentes do que as pontes de veia safena. Este fato, aliado à possibilidade de se revascularizar o território das artérias coronária direita e circunflexa, ampliou a indicação do emprego dessa técnica. Atualmente, em mais da metade dos pacientes operados no Serviço, tem-se empregado pelo menos uma artéria mamária. Gostariamos, ainda, de enfatizar que alguns estudos realizados já demonstraram que o controle dos fatores de risco, principalmente o colesterol, o tabagismo e a hipertensão arterial, é capaz de reduzir o número de obstruções de pontes de safena. 